\title{
An Asymptotic Solution for Washcoat Pore Diffusion in Catalytic Monoliths
}

\author{
Edward J. Bissett
}

Received: 9 December 2014 / Revised: 18 December 2014 / Accepted: 5 January 2015 / Published online: 29 January 2015

(C) Springer SIP, AG 2015

\begin{abstract}
When modeling monolithic exhaust aftertreatment reactors, washcoat pore diffusion resistance is often neglected with satisfactory results, but interest in the effect remains, especially for dual layer catalysts where the intention is to explicitly exploit the effect, for example, to enhance selectivity. To bring analyses that include washcoat pore diffusion resistance into convenient and everyday usage, an asymptotic solution based on small dimensionless washcoat pore diffusion resistance is derived and presented herein. When this solution is integrated within a common formulation for modeling monolithic exhaust aftertreatment reactors, it solves the leading-order reaction-diffusion equations within the washcoat analytically so the computational burden associated with discretizing and numerically solving along the washcoat thickness dimension is avoided. No further ad hoc approximations are required; in particular, the full complexity and nonlinearity of the reaction system is preserved. Catalytic surface coverages and dual layer catalysts are included. The method can be implemented in a manner that generalizes but is similar to the more standard solution method for no pore diffusion resistance. Although the asymptotic solution cannot substitute for the full solution in all cases, we argue that this asymptotic regime includes the most practical applications.
\end{abstract}

Keywords Washcoat · Diffusion · Modeling · Asymptotic · Monolith · Catalytic

\section{Nomenclature \\ a Vector of dimensionless active site densities \\ $B i \quad$ Biot number, $D_{i n v} K$ \\ c Concentration $\mathrm{mol} / \mathrm{m}^{3}$}

\section{E. J. Bissett $(\square)$}

Gamma Technologies, 601 Oakmont Lane, Suite 220,

Westmont, IL 60559, USA

e-mail: e.bissett@gtisoft.com
$C_{p g} \quad$ Dimensionless heat capacity of gas

$D_{\text {eff }} \quad$ Diagonal matrix of effective diffusivities $\mathrm{m}^{2} / \mathrm{s}$

$D_{h} \quad$ Hydraulic diameter of channel $\mathrm{m}$

$D_{i n v} \quad$ Diagonal matrix of dimensionless pore diffusion resistances, see Eq. (41)

$D_{r} \quad$ Reference diffusivity, see Eq. (42) $\mathrm{m}^{2} / \mathrm{s}$

$f_{w c} \quad$ Volume fraction of washcoat within reactor

$h \quad$ Dimensionless heat transfer coefficient

$K \quad$ Diagonal matrix of dimensionless mass transfer coefficients

$L \quad$ Length of reactor $\mathrm{m}$

nrct Total number of reactions

$q \quad$ Scaled site association matrix, see Eq. (19)

$R \quad$ Vector of dimensionless species mass rates

$r \quad$ Vector of dimensionless reaction rates

$s \quad$ Scaled stoichiometric matrix

$S \quad$ Specific area of reactor (channel area/reactor volume) $\mathrm{m}^{-1}$

$t \quad$ Dimensionless time

$T_{g} \quad$ Dimensionless mixing cup temperature of channel gas

$T_{s} \quad$ Dimensionless washcoat temperature

$V \quad$ Reactor volume $\mathrm{m}^{3}$

$W \quad$ Dimensionless mass flow rate into reactor

$W_{r} \quad$ Reference mass flow rate used for nondimensionalization $\mathrm{kg} / \mathrm{s}$

$x \quad$ Dimensionless position through the washcoat

$z \quad$ Dimensionless axial position through reactor

$\delta \quad$ Effective washcoat thickness $\mathrm{m}$

$\Delta H \quad$ Dimensionless vector of reaction enthalpies

$\theta \quad$ Vector of scaled surface coverages

$\lambda_{s} \quad$ Dimensionless thermal conductivity of wall

$\rho_{s} \quad$ Density of gas within washcoat $\mathrm{kg} / \mathrm{m}^{3}$

$\sigma \quad$ Scaled stoichiometric matrix for coverage, see Eq. (18)

$\phi \quad$ Thiele modulus, see Eq. (36)

$\psi_{s} \quad$ Dimensionless effective heat capacity of reactor

$\omega \quad$ Vector of scaled mass fractions in the washcoat

$\omega_{g} \quad$ Vector of scaled mass fractions of channel gas 
$\omega_{r} \quad$ Vector of reference mass fractions used for scaling

$\omega_{s} \quad$ Vector of scaled washcoat surface mass fractions, $\omega(x=0)$

\section{Introduction}

In modeling automotive exhaust aftertreatment reactors, both reactions on the catalytic sites within the washcoat and transport of exhaust gas to these sites are known to be important and capable of affecting the net species rates of interest. Longstanding common practice has been to include the external mass transfer within the standard 1-D approach to monolithic channels using mass transfer coefficients to capture the rate of diffusion within the monolith channels. The internal mass transfer resistance, that is, from diffusion within the porous washcoat, is often neglected for either or both of two main reasons:

- The washcoat properties (effective diffusivities), in particular, the small thickness of the washcoats (tens of microns), do not support large concentration differences.

- The computational burden of including the reaction-diffusion problem within the washcoat at each axial location is excessive.

Decades of successful modeling of exhaust aftertreatment reactors without pore diffusion resistance suggests that this is a reasonable approximation for many applications. Nevertheless, interest in washcoat pore diffusion resistance has remained, at a minimum for applications of washcoats with dual layers. These dual layer catalysts can be specifically chosen to exploit the different internal mass transfer rates within the layers. A conservative overall assessment would be that pore diffusion resistance is an effect that is:

- Probably important for specific applications (for example, dual layer catalysts)

- Worth estimating even if not judged important

- Computationally expensive if the resulting washcoat diffusion-reaction equations are coupled with the 1-D monolith reactor equations

In this research, we propose an intermediate approach that captures the major effect of pore diffusion resistance without the computational expense. Our method is to obtain an asymptotic solution of the governing equations for small pore diffusion resistance. This solution has the potential to bring previously burdensome analyses into the routine realm, especially for new catalyst formulations meant to exploit washcoat pore diffusion resistance such as dual layer catalysts. Some key points to note about this solution:
- Analytical solution of the diffusion-reaction system within the washcoat avoids the associated computation expense.

- It reduces to the limiting case of the standard 1-D approach without pore diffusion resistance

- The calculation includes the first-order terms for small pore diffusion resistance, so it captures the dominant asymptotic effects.

In the Discussion section, we address the qualitative and quantitative issues regarding the accuracy and applicability of this solution, and we contrast it to existing asymptotic solutions based on the Thiele modulus. For example, when the washcoat pore diffusion resistance is small, but a reactant effective rate constant becomes large at high temperatures, then the local species rate becomes transport limited and the washcoat concentrations become insignificant. Finally, we present sample results for a simple case that can easily be compared with the alternative numerical solution for the reaction-diffusion equations in the washcoat, and we present a comparison with published data from a dual layer catalyst with complex kinetics. These examples are intended to demonstrate the applicability and utility of the method when effective diffusivities are chosen in the intended and practical range around $10^{-6} \mathrm{~m}^{2} / \mathrm{s}$ or larger.

\section{Single Layer, No Coverages}

\subsection{Formulation}

To simplify and follow best modeling practices, we work with the dimensionless form of the governing equations. To initially present the idea in the simplest possible setting, we first restrict to a single washcoat layer with no coverages and will later relax these restrictions. Many global reaction rates, including most over noble metal sites, are written without coverages, so this is not a trivial case.

$\psi_{s} \frac{\partial T_{s}}{\partial t}=\frac{\partial}{\partial z}\left(\lambda_{s} \frac{\partial T_{s}}{\partial z}\right)+h\left(T_{g}-T_{s}\right)-\sum_{j=1}^{n r c t} a_{j} \Delta H_{j} \bar{r}_{j}$

$W C_{p g} \frac{\partial T_{g}}{\partial z}=h\left(T_{s}-T_{g}\right)$

$W \frac{\partial \omega_{g}}{\partial z}=K\left(\omega_{s}-\omega_{g}\right)$

$\frac{\partial \omega}{\partial x}=D_{i n v} K\left(\omega_{s}-\omega_{g}\right), \quad x=0$ 
$\frac{\partial^{2} \omega}{\partial x^{2}}=-D_{\text {inv }} R, \quad 0<x<1$

$\frac{\partial \omega}{\partial x}=0, \quad x=1$

The Nomenclature section describes the quantities appearing in these equations. Beyond this, several points deserve emphasis or clarification. In this nondimensionalization, the axial location, $z$, has been scaled by the reactor length, and the time, $t$, has been scaled by a warm-up time that balances the transient term on the left-hand side of Eq. (1) with its drivers on the right (typically about $1 \mathrm{~s}$ ). The location within the washcoat, $[0<x<1]$, measured from the gas interface, is scaled by an effective thickness,

$\delta=f_{w c} / S$,

that we prefer as a small correction to a point-wise measured thickness since it unambiguously includes all the washcoat volume, including the "corners". Temperatures are scaled by a fixed reference temperature (for example, $600 \mathrm{~K}$ ), and each component of the species mass fraction vector, $\omega$, is scaled by its application-specific magnitude. The main purpose of the nondimensionalization (including scaling of dimensionless dependent variables like mass fractions or, later, coverages) is to create $O(1)$ variables and $O(1)$ coefficients in the governing equations for all dominant terms. Equations (3)-(6) are vector equations, and $K$ and $D_{i n v}$ are diagonal matrices with the same number of nonzero elements as $\omega . \omega_{s} \equiv \omega(0)$ is introduced as a convenience, not as an additional unknown. As the details of the nondimensionalization are not critical to the result, readers should feel free to use their own nondimensionalization that respects the particular points of this paragraph. Demanding $O(1)$ variables and $O(1)$ coefficients, in particular, those pertaining to the species rates and the concentrations, may impose additional restrictions on the applicability of the asymptotic solution, as will be discussed later.

The species rates are related to the reaction rates via a stoichiometric matrix.

$R=s r$

This follows the convention that products have positive stoichiometric coefficients, and reactants have negative. The washcoat temperature, $T_{s}$, is assumed constant across the washcoat because of conduction across the thin layer. Therefore, the rate in Eq. (1) is the integrated rate across the washcoat, which is a simple average here because the integration length is 1 . Finally, we assume an ideal gas with a constant pressure to obtain the gas density because the momentum-driven pressure differences are small compared to the pressure itself. The density is used to convert the species mass fractions to concentrations (moles $/ \mathrm{m}^{3}$ in the dimensional setting) appropriate for evaluation of the reaction rates. As this part of the calculation is not explicitly a part of our manipulations, we skip further details for handling pressure, density, momentum, and the equation of state. The result is a dimensionless form of the quasi-static governing equations for monolithic aftertreatment reactor modeling that is sometimes called (1+1)-dimensional: 1 dimension for the axial location plus a subsidiary dimension for the location through the washcoat at each such axial location.

\subsection{Motivation}

An important preliminary step in preparation for the derivation is to integrate the reaction-diffusion Eq. (5) across the washcoat and use its boundary conditions for the flux to obtain

$K\left(\omega_{s}-\omega_{g}\right)=\bar{R}(\omega) \equiv \int_{0}^{1} R(\omega(x)) d x$.

This result is true independently of any assumptions regarding the magnitude of the pore diffusion resistance.

Now, consider the limiting case of no diffusion resistance, $D_{i n v}=0$. Equation (5) then states that $\omega$ can be at most linear in $x$, but Eq. (6) (as well as Eq. (4) in this limit) restricts this linear function to a constant. Therefore, $\omega=\omega(0)=\omega_{s}$, a constant. Since the rates depend only upon $T_{s}$ and $\omega$, which are independent of $x$, the rates, $r$ and $R$ are independent of $x$, $\bar{R}(\omega)=R\left(\omega_{s}\right)$, and Eq. (9) becomes the familiar requirement, $K\left(\omega_{s}-\omega_{g}\right)=R\left(\omega_{s}\right)$, that is generally imposed directly from physical arguments in the absence of pore diffusion resistance. We will refer to this as the standard problem and its solution as the standard solution. It provides a legitimate, but trivial, solution of the reaction-diffusion problem in this extreme case of $D_{i n v}=0$. Since it only requires evaluation of the rates at a single mixture, $R\left(\omega_{s}\right)$, for each axial location, this standard problem is tractable for quite large systems of nonlinear reactions without further approximations and has proven its value modeling exhaust aftertreatment reactors for decades. Our interest here is to maintain this standard of generality for the systems of nonlinear reactions while extending solutions of the washcoat reaction-diffusion problem beyond $D_{i n v}=0$.

$D_{i n v}$ is the inverse of the diagonal matrix of dimensionless effective diffusivities. As the effective diffusivities go to infinity, $D_{i n v} \rightarrow 0$. For our purposes, it is easier to consider small diffusion resistances, $D_{i n}$, than large effective diffusivities. In particular, the preceding paragraph shows that the standard problem is contained within the more general formulation as $D_{i n v} \rightarrow 0$. To put this into the asymptotic framework, we identify the small parameter, $\left\|D_{i n v}\right\|$, for any convenient norm, and formally recognize the standard solution as the zeroth-order solution of the general formulation, since it holds when 
$\left\|D_{i n v}\right\|=0$. From this viewpoint, we will capture the most important effect of pore diffusion resistance after extending this analysis to include first-order, $O\left(\left\|D_{i n v}\right\|\right)$, terms of the solution. This derivation and its resulting computation become tractable largely because we freely drop even higher-order $O\left(\left\|D_{i n v}\right\|^{2}\right)$ terms. Note that actually solving the standard problem is still formidable as a system of coupled partial differential and algebraic equations with a potentially large number of species and nonlinear reaction rates. The intended applications are those routinely encountered by advanced commercial aftertreatment modeling software where the reaction rates, $r(\omega)$, involve dozens of species and dozens of nonlinear reaction rates that for practical reasons must be evaluated and solved as given. The details of solving the standard problem are outside our current scope. The asymptotic solution described herein is no simpler or easier, but our aim here is to show that, after the conceptual hurdles have been cleared, it is not appreciably more difficult nor does it require approximating or modifying the reaction rates from their full original nonlinear forms.

\subsection{Asymptotic Solution}

To extend the solution to an additional order, an additional term from all of the governing equations that were used to generate the zeroth-order standard solution is generally required. The key will be Eq. (9), and we already know that the zeroth term of the species rate, $R$, is constant because the zeroth term of its argument, $\omega$, is constant, although we do not yet know the constant explicitly. We can expand $R(\omega)$ about any arbitrary constant vector, which we temporarily and suggestively name $\bar{\omega}$, as follows.

$R(\omega)=R(\bar{\omega})+\left.\frac{\partial R}{\partial \omega}\right|_{\bar{\omega}}(\omega-\bar{\omega})+O\left(\left\|D_{i n v}\right\|^{2}\right)$

The $O\left(\left\|D_{i n v}\right\|^{2}\right)$ error term captures our expectation from the Motivation section that $\omega-\bar{\omega}=O\left(\left\|D_{i n v}\right\|\right)$. The Jacobian in the expression is also constant in $x$ because it is evaluated at $\omega=\bar{\omega}$ (and $T_{s}$ is constant). Therefore, if we indeed take

$\bar{\omega}=\int_{0}^{1} \omega(x) d x$

and average Eq. (10), the term with the Jacobian will drop out, resulting in

$\bar{R}(\omega)=\bar{R}+O\left(\left\|D_{i n v}\right\|^{2}\right)$,

where we now introduce, when convenient, the notation, $\bar{R} \equiv R(\bar{\omega})$. This notation is intended to highlight the distinction between the average of the rate and the rate of the average while still remaining compact. This simple but important result is accurate including first-order terms, requires us to know $\bar{\omega}$ through first-order terms, and eliminates the need to introduce a Jacobian to extend Eq. (9) to higher order. Equation (10) also yields the simpler point-wise result that

$R(\omega(x))=\bar{R}+O\left(\left\|D_{\text {inv }}\right\|\right)$

Now Eq. (5) can be solved through first-order terms, while assigning the resulting constants of integration using Eqs. (6) and (11):

$\omega(x)=\bar{\omega}+D_{\text {inv }} \bar{R}\left[\frac{1}{6}-\frac{1}{2}(1-x)^{2}\right]+O\left(\left\|D_{i n v}\right\|^{2}\right)$,

After $\bar{\omega}$ is known, this equation provides the first-order solution to the reaction-diffusion equations in the washcoat. The overall magnitude of the species are given by $\bar{\omega}$, which here must contain both zeroth and first-order terms to provide the same accuracy to $\omega(x)$ itself. The spatial variation is smaller, $O\left(\left\|D_{i n v}\right\|\right)$, given by the quadratic in Eq. (14). From this, $\omega_{s}=\omega(0)$ can be evaluated:

$\omega_{s}(\bar{\omega})=\bar{\omega}-D_{i n v} \bar{R} / 3+O\left(\left\|D_{i n v}\right\|^{2}\right)$.

We use this in Eq. (9) to obtain the required nonlinear system that determines $\bar{\omega}$ :

$K\left(\omega_{s}(\bar{\omega})-\omega_{g}\right)=R(\bar{\omega})+O\left(\left\|D_{i n v}\right\|^{2}\right)$

For a practical implementation, the computation can be organized similarly to the standard problem. $\bar{\omega}$ replaces $\omega_{s}$ as the fundamental variable describing the washcoat species while $\omega_{s}(\bar{\omega})$ is a known function from Eq. (15) rather than the unknown itself. This adjustment is made to the two coupled equations for the species in the channel and washcoat, Eqs. (3) and (16).

$\bar{r}(\omega)=r(\bar{\omega})+O\left(\left\|D_{i n v}\right\|^{2}\right)$

holds for the same reason as $R$ and is used in Eq. (1) and to evaluate $R(\bar{\omega})=\operatorname{sr}(\bar{\omega})$. All $O\left(\left\|D_{i n v}\right\|^{2}\right)$ terms may be dropped in computing the necessary quantities for the first-order solution. When organized this way, only one reaction rate evaluation and no Jacobian is required to solve the reaction-diffusion equations at each axial location even though average reaction rates are required through first-order terms. The required first-order accuracy of Eqs. (12) and (17) necessitates the use of $\bar{\omega}$ as fundamental unknowns. Other constants, for example $\omega_{s}$, could be used in principle to express the asymptotic solution in place of Eq. (14), but they still must be related back to $\bar{\omega}$, which is essential for the rate evaluations. 
The approximation is wholly based upon the asymptotics of pore diffusion resistance. The linearization in Eq. (10) is implied by the small pore diffusion resistance solution itself and is not an additional assumption. It is used as an intermediate on the way to the nonlinear Eq. (16). Nowhere is it necessary to further simplify the rate expressions through ad hoc linearizations, effectiveness factors, or the like.

\section{Single Layer, with Coverages}

The concepts of the previous section carry through to this case. Here, we discuss the additional complications introduced by the presence of coverages. These arise within the governing equations for the coverages and from the dependence of the reaction rates upon the coverages.

We make the additional assumptions that any nontrivial $x$ variation of the initial coverages, if present, is small, $O\left(\left\|D_{i n v}\right\|\right)$ at most. This is a minor restriction, since the coverages for practical modeling exercises are usually initialized with a constant and seldom known in enough detail to assign even a nontrivial axial distribution, so a further nontrivial initial distribution through the washcoat layer is unlikely to be needed or even known to the modeler.

The defining governing equations for the coverages are local. Any individual coverage, indexed by $k$ within the coverage vector, will commonly satisfy a rate equations given by the stoichiometry of the reactions:

$\frac{\partial \theta_{k}}{\partial t}=\sum_{j=1}^{n r c t} \sigma_{k j} r_{j}$

If the vacant sites are included in the coverage vector, there are also linear algebraic equations capturing the requirement that the sum of all unscaled coverages on each site is 1 :

$\sum_{k} q_{i k} \theta_{k}=1$

A modeler may choose to use one or more of the equations in Eq. (19) to replace the same number from Eq. (18) for the same site. This is a matter of preference and modeling style not important to this argument. The point is that these local equations hold at each location $(x, z)$. Below, we verify that these additional equations may be included within the asymptotic solution.

First consider Eq. (18) at zeroth order at a particular axial location. The zeroth-order rates here depend upon the coverages themselves, the zeroth-order washcoat species which are known to be constant in $x$ from the small $\left\|D_{i n v}\right\|$ asymptotics above, and the constant $T_{s}$. Therefore, since each coverage is initialized independently of $x$, they will continue to evolve independently of $x$ at zeroth order, although the first-order corrections in general will not. That is, $\theta=\bar{\theta}+O\left(\left\|D_{i n v}\right\|\right)$, so that an expansion like Eq. (10) gives

$$
\begin{aligned}
r(\omega, \theta)= & r(\bar{\omega}, \bar{\theta})+\left.\frac{\partial r}{\partial \omega}\right|_{\bar{\omega}, \bar{\theta}}(\omega-\bar{\omega})+\left.\frac{\partial r}{\partial \theta}\right|_{\bar{\omega}, \bar{\theta}}(\theta-\bar{\theta}) \\
& +O\left(\left\|D_{\text {inv }}\right\|^{2}\right)
\end{aligned}
$$

Averaging, we recover Eq. (17) in the form

$\bar{r}(\omega, \theta)=r(\bar{\omega}, \bar{\theta})+O\left(\left\|D_{i n v}\right\|^{2}\right)$,

and after multiplication by $\sigma$, Eq. (12) is recovered as

$\bar{R}(\omega, \theta)=\bar{R}+O\left(\left\|D_{i n v}\right\|^{2}\right)$,

where the definition of $\bar{R}$ is generalized here to

$\bar{R} \equiv R(\bar{\omega}, \bar{\theta})$.

From this point, the derivation of the washcoat species proceeds as before, leading to Eqs. (14)-(16), with $\bar{R}$ now a function of both $\bar{\omega}$ and $\bar{\theta}$.

The above expressions require a first-order accurate solution for $\bar{\theta}$ but not $\theta$. The required equations come from averaging Eqs. (18) and (19), using the first-order accurate result of Eq. (21):

$\frac{\partial \bar{\theta}_{k}}{\partial t}=\sum_{j=1}^{n r c t} \sigma_{k j} r_{j}(\bar{\omega}, \bar{\theta})+O\left(\left\|D_{i n v}\right\|^{2}\right)$.

$\sum_{k} q_{i k} \bar{\theta}_{k}=1$

This asymptotic solution is capable of providing the first-order accurate average coverages, but any small, $O\left(\left\|D_{i n v}\right\|\right)$, variations in the $x$-direction are neither needed nor provided by the solution.

\section{Dual Layers, with Coverages}

Again, we emphasize the new considerations for dual layers while keeping repetition of earlier results to a minimum. Many of our variables are specific to each layer. For example, each layer has its own independent set of reaction rates and coverages, although the species are common to both. That is, if a species reacts in only one layer, it at least diffuses through the other. We use a parenthetical superscript to denote a variable specific to a layer, for example, $r^{(1)}, \bar{\theta}^{(2)}$, and so forth. We use the above results that remain true for layer 1 as if they were 
originally written with the parenthetical superscript, (1) or (2), without further elaboration.

For the nondimensionalization of layer 2, we again use an effective thickness, $\delta^{(2)}=f_{w c}^{(2)} / S$, but map the second layer to the interval $[1,2]$, adjacent to layer 1 . An average quantity for layer 2 is an integral of the quantity from $x=1$ to 2 .

Regarding the governing equations, Eq. (1) here contains additional contributions to the effective heat capacity and thermal conductivity, $\psi_{s}$ and $\lambda_{s}$, weighted according to their volume fractions. The heat release term in this equation must now sum over the reactions in both layers.

For the species equations, Eqs. (4) and (5) still hold for $0 \leq x$ $<1$, but here, we impose the necessary continuity at $x=1$ and move the no-flux boundary condition to $x=2$ :

$\omega(1-)=\omega(1+)$

$\left[D_{i n v}^{(1)}\right]^{-1} \frac{\partial \omega}{\partial x}(1-)=\left[D_{i n v}^{(2)}\right]^{-1} \frac{\partial \omega}{\partial x}(1+)$

$\frac{\partial^{2} \omega}{\partial x^{2}}=-D_{i n v}^{(2)} R^{(2)} \quad 1<x<2$

$\frac{\partial \omega}{\partial x}=0 \quad x=2$

Integrating over [0,2], using the boundary and flux continuity conditions, yields the generalization of Eq. (9)

$K\left(\omega_{s}-\omega_{g}\right)=\bar{R}^{(1)}(\omega)+\bar{R}^{(2)}(\omega)$.

The arguments leading to Eqs. (21) and (22) apply as before in each layer, as well as the coverage equations, Eqs. (24) and (25). In particular, the argument that $\omega$ is constant to leading order in layer 2 proceeds as before. Then flux continuity at $x=$ 1 implies $\omega$ is constant to leading order in layer 1 .

The differential equations for the species profiles in the layers can again be readily solved since their right-hand sides are constant to the required order of approximation. The four constants of integration are satisfied by flux continuity at $x=1$, zero flux at $x=2$, and the two conditions that $\bar{\omega}^{(1)}$ and $\bar{\omega}^{(2)}$ are indeed the averages over their respective layers. Equivalently, we can consider the two flux conditions as eliminating two of the constants of integration and consider the averages, $\bar{\omega}^{(1)}$ and $\bar{\omega}^{(2)}$, to be the two remaining constants of integration.

$$
\begin{aligned}
\omega= & \bar{\omega}^{(1)}+D_{i n v}^{(1)} \bar{R}^{(1)}\left[\frac{1}{6}-\frac{1}{2}(1-x)^{2}\right]+D_{i n v}^{(1)} \bar{R}^{(2)}\left(x-\frac{1}{2}\right) \\
& +O\left(\left\|D_{i n v}\right\|^{2}\right), \quad 0<x \leq 1
\end{aligned}
$$

$$
\omega=\bar{\omega}^{(2)}+D_{i n v}^{(2)} \bar{R}^{(2)}\left[\frac{1}{6}-\frac{1}{2}(2-x)^{2}\right]+O\left(\left\|D_{i n v}\right\|^{2}\right), \quad 1<x \leq 2
$$

The final conditions to determine the washcoat species are species continuity at $x=1$ and the first-order approximation to Eq. (30), which implicitly captures the boundary condition at $x=0$.

$$
\begin{aligned}
& K\left[\omega_{s}\left(\bar{\omega}^{(1)}, \bar{\omega}^{(2)}\right)-\omega_{g}\right]-R^{(1)}\left(\bar{\omega}^{(1)}\right)-R^{(2)}\left(\bar{\omega}^{(2)}\right) \\
& =O\left(\left\|D_{\text {inv }}\right\|^{2}\right)
\end{aligned}
$$

$$
\begin{aligned}
& \bar{\omega}^{(2)}-\bar{\omega}^{(1)}-\frac{D_{i n v}^{(1)}}{6} R^{(1)}\left(\bar{\omega}^{(1)}\right)-\left(\frac{D_{i n v}^{(1)}}{2}+\frac{D_{i n v}^{(2)}}{3}\right) R^{(2)}\left(\bar{\omega}^{(2)}\right) \\
& =O\left(\left\|D_{i n v}\right\|^{2}\right)
\end{aligned}
$$

These are the coupled nonlinear equations that must be solved numerically for $\bar{\omega}^{(1)}$ and $\bar{\omega}^{(2)}$ after again dropping the $O\left(\left\|D_{\text {inv }}\right\|^{2}\right)$ terms. They are the generalization of Eq. (16) to dual layers. In Eq. (33), $\omega_{s}\left(\bar{\omega}^{(1)}, \bar{\omega}^{(2)}\right)$ is given by

$\omega_{s}=\bar{\omega}^{(1)}-\frac{D_{i n v}^{(1)} \bar{R}^{(1)}}{3}-\frac{D_{i n v}^{(1)} \bar{R}^{(2)}}{2}+O\left(\left\|D_{i n v}\right\|^{2}\right)$

\section{Discussion}

\subsection{Accuracy}

The solution proposed here is a two-term asymptotic expansion using the small parameter, $\left\|D_{i n v}\right\|$. For practical numerical computation, we have structured it so that both terms are calculated together. The zeroth-order term agrees with the solution for the standard problem when pore diffusion resistance is completely negligible. The success of past standard solutions suggests that including the additional first-order terms will be accurate enough in most circumstances. Asymptotic solutions achieve their accuracy through the magnitude of their controlling parameter (here, $\left.\left\|D_{i n v}\right\|\right)$, not necessarily by including more terms. We are fortunate that the first-order terms here are available with relatively little additional computational effort, require no ad hoc simplification of the reaction rate expressions, and result in simple analytical solutions of the differential equations for the washcoat species as functions of $x$. However, these advantages rapidly disappear if additional terms in the expansion are attempted, so this extension is not recommended. 
To go beyond the qualitative argument above, quantitative accuracy depends upon the magnitude of $\left\|D_{\text {inv }}\right\|$, assuming the other dimensionless quantities important to the solution remain $O(1)$. An excellent summation of the appropriate expectations for this assessment is captured in the following quotation from a classic text on the subject [1, p. 124]. In this quote, $x$ is the asymptotic parameter, that is, $\left\|D_{i n v}\right\|$ in our case: “... for any given problem we can never really know a priori whether or not asymptotic analysis will give good numerical results at a fixed value of $x$. However, experience has shown that asymptotic methods nearly always give spectacularly good results." To elaborate, quantitative accuracy of an asymptotic expansion is not a property of the expansion alone - it formally requires additional knowledge of the full solution or at least the next term in the expansion. Although it is futile to demand a priori bounds on the error of the approximation, we can often expect far better results than apparently justified by the magnitude of $\left\|D_{\text {inv }}\right\|$ alone.

\subsection{Magnitude of $D_{i n v}$}

With that said, what is a typical magnitude of $\left\|D_{\text {inv }}\right\|$ ? Because this is a general method, we have avoided tying it to a particular nondimensionalization; the reader should feel free to follow their preferences. Following estimates detailed in Appendix 1 , we find $\left\|D_{i n v}\right\|$ to be of the order of 0.1 or smaller, when $D_{\text {eff }, i}$ are taken in the common range of $10^{-6} \mathrm{~m}^{2} / \mathrm{s}$ or larger. In a sense, this range is ideal since it is small enough to justify $\left\|D_{i n v}\right\| \ll 1$ for mainstream applications without the effect becoming uninterestingly small. Such estimates should not discourage a reader from attempting the asymptotic solution even in more extreme applications because of the above general comments regarding unexpected success of the asymptotics. The results can always be checked a posteriori that the changes in species across the washcoat are at least somewhat smaller than the species magnitudes themselves, which is the fundamental feature supporting the asymptotic solution.

Estimates of washcoat effective diffusivities are difficult and somewhat controversial. Our purpose here is to provide a new tool to study the effects of pore diffusion resistance, not to weigh in on the question of magnitudes, although the magnitude of effective diffusivities obviously affects applicability. One of many possible entries into this extensive literature is the work of Hayes and others, $[2,3]{ }^{1}{ }^{1}$ who have worked to measure $D_{\text {eff: }}$ Certainly, if modelers assume sufficiently small effective diffusivities, they can invalidate the asymptotic solution. Therefore, this asymptotic solution is not intended for such extreme cases, or even for some modeling studies where

\footnotetext{
${ }^{1}$ It is of some interest to note that a number of authors use [2] to reference a measured $D_{\text {eff }}$ as low as $1 \times 10^{-7} \mathrm{~m}^{2} / \mathrm{s}$. In [3], the authors from the same group as [2] explain that the sample in question contained no macropores, resulting in $D_{\text {eff }}$ about an order of magnitude smaller than the more typical commercial catalytic converter used in the later reference.
}

the magnitude of effective diffusivity is chosen intentionally to ensure the onset of large pore diffusion limitations.

\subsection{Comparison with Thiele Modulus}

A reader familiar with the long history of diffusion and reaction within porous catalysts might question why we do not/ cannot use the traditional asymptotic approach based on Thiele modulus, which measures the ratio of effective rate constant to effective diffusivity. In this section, we address this question and explain the above asymptotic solution relative to this traditional viewpoint. The main purpose here is to aid readers accustomed to analyzing the traditional way, but these observations may prove valuable independently. It is sufficient and therefore clearer to make these qualitative points considering the simpler case of a single layer, but the extension to dual layers is not difficult. To preview, some main points of comparison are listed in Table 1 in an oversimplified form to be elaborated as necessary in the remainder of this section. In the table heading, $\phi$ is the Thiele modulus, defined for each reactant species as

$\phi_{i}^{2}=-D_{i n v, i} k_{i}$,

where $k_{i}$ is its rate constant (among a number of possible definitions in the nonlinear setting, our preference is Eq. (39)). When $k_{i}=O(1)$ as formally assumed in this paper, $\left\|D_{\text {inv }}\right\| \ll 1$ will imply $\phi_{i}^{2} \ll 1$. The Thiele modulus approach also supports the alternative asymptotic limit of $\phi^{2} \gg 1$, which is not directly relevant to the limit of this paper.

Because the Thiele modulus is defined in terms of a rate constant, its use presumes the foremost feature of the reaction rates is the relative magnitude of the species rates compared to their corresponding species. By contrast, the analysis of this paper avoids any dependence upon any rate constant and rather makes the assumption that the rates themselves are $O(1)$, although we will discuss below how this assumption is less restrictive than might first appear for our applications of interest. The history, and hence the legacy, of the Thiele modulus approach, as described in detail for example in [4], began with the application to a single species with a single linear reaction rate and the Dirichlet boundary conditions for given $\omega_{s}$. For this case, the Thiele modulus alone can be used to characterize all possible solutions. These resulting solutions have inspired generalizations too numerous to detail here, but the more the application departs from this simple ideal case, the more assumptions and/or approximations must be added to retain meaningful results. With no attempt to be exhaustive, several examples of these assumptions and/or approximations are given below:

- When the reactions are not linear, the "rate constant" obviously cannot be constant. Local conditions may preclude the confident application of any local linearization, 
Table 1 Comparison with small Thiele modulus

\begin{tabular}{|c|c|c|}
\hline & $\left\|D_{i n v}\right\| \ll 1$ & $\phi^{2} \ll 1$ \\
\hline 1 & Transport-based & Reaction-based \\
\hline 2 & $\begin{array}{l}\text { Originated with solutions for no diffusion resistance } \\
\text { for large complex systems }\end{array}$ & $\begin{array}{l}\text { Originated with solutions for diffusion-reaction of a single species } \\
\text { with single linear rate }\end{array}$ \\
\hline 3 & Restricts transport, $\left\|D_{i n v}\right\| \ll 1$ & No transport restriction if $\phi^{2}$ varied via rate constants \\
\hline 4 & No restriction on system $R(\omega)$ & $R(\omega)$ can be approximated by forms using rate constants \\
\hline 5 & Fully nonlinear & Requires linear, single-species results for some key steps \\
\hline 6 & $\begin{array}{l}\text { Provides explicit solution of original reaction-- } \\
\text { diffusion system }\end{array}$ & $\begin{array}{l}\text { Replaces diffusion terms with algebraic results from simpler idealized } \\
\text { systems (e.g., effectiveness factor or internal Sherwood number) }\end{array}$ \\
\hline 7 & Explicit solution generalizes to dual layers & $\begin{array}{l}\text { No dual layer generalization since not present in simpler systems used } \\
\text { to replace diffusion terms }\end{array}$ \\
\hline 8 & All approximations derived from $\left\|D_{i n v}\right\| \ll 1$ & Many additional problem-specific approximations \\
\hline 9 & Biot number, $B i=O\left(\left\|D_{i n v}\right\|\right) \ll 1$ & Usually $B i=O(1)$ or larger \\
\hline 10 & Priority is reactor output via $\omega_{g}(z)$ & Priority is washcoat concentration $\omega(x)$ \\
\hline
\end{tabular}

subject to individual analysis of each condition of interest. At minimum, there is ambiguity in the assignment of the rate constant for purposes of the Thiele modulus, and this is a major source of the additional assumptions and/or approximations mentioned above. For one example, in a recent publication, the rate constants are first posed as a matrix of partial derivatives evaluated at the catalyst surface (Eq. (22) of [5]), then assumed to be diagonal with the partial derivatives replaced by the ratio of species rate to species, and the concentration for evaluation is then approximated by the channel gas cup-mixing average (Eq. (25) of [5]), which can be quite different from the washcoat concentrations.

- Current modern applications require dozens of reactions and species, some of which are products (for example, $\mathrm{NO}_{2}$ or $\mathrm{NH}_{3}$ may be absent in the inlet gas but are desired products for some intermediate exhaust aftertreatment reactors). Products are not naturally addressed with the Thiele modulus approach because their production rates only depend directly (that is, excluding dependence through inhibition terms) upon other reactants, so any reasonable assignment of a ratio for this species will not represent a meaningful rate constant.

- A common, but not universal, aspect of the Thiele modulus approach is to generate an effectiveness factor. This factor then reduces the relevant species rate from the more readily available rate evaluated at $\omega_{s}$, for example in [6] in the context of a variety of modeling approaches. Of course, there is a significant approximation in generalizing the effectiveness factor-Thiele modulus relationship obtained from considerably simpler systems to the more complex system at hand. However, the additional practical difficulty that also bears mentioning is that the effectiveness factor is applied to the species rates, but these complex systems are rather defined by the reaction rates.
Although (8) allows the species rates to be directly obtained from the reaction rates, the reverse is not generally true. In general, the stoichiometric matrix, $s$, cannot be inverted, so additional assumptions/approximations are involved with the application of the effectiveness factors to ensure consistency between the reaction rates and their evaluation for the coverages in Eq. (18). The reference [6] describes one possible assumption in which the effectiveness factor for a single species is applied throughout.

- An alternative use of the Thiele modulus which avoids effectiveness factors is to generate an "internal mass transfer coefficient" as developed in a series of papers partially represented by [7-10]. In very simple terms, this approach approximates the effect of washcoat diffusion within the given reaction-diffusion problem with that of a simpler linear problem at the extremes of Thiele modulus and posits a smooth transition between the extremes [8]. The results of this approximation are introduced into the original problem of interest using an internal mass transfer coefficient. A major qualitative difference from the more usual external mass transfer coefficient is that this internal coefficient is derived from a simplified approximate system that still contains the reactions and must capture their coupled effects. The species rates are considered averages for use in Eq. (9). Beyond these approximations, there is a more subtle but important assumption made in this approach at the key step when the average rate is replaced with the rate of the average, Eq. (12). In contrast, recall that this result can be derived, not assumed, for the asymptotic limit $\left\|D_{\text {inv }}\right\| \ll 1$ of this paper because the washcoat profiles are approximately flat. Without $\left\|D_{i n v}\right\| \ll 1$, this result requires an additional assumption at best when working with internal mass transfer coefficients, and is highly questionable for the rapidly-varying washcoat profiles of large Thiele modulus at worst. In the cited papers 
for example, the spatially variations of the concentrations are assumed small compared to their averages without further comment in [9] and [10]. In [8], the equivalent of the second-order terms of Eq. (12) are ignored as higher order. The small parameter that would represent the magnitude of these higher-order terms is not explicit, but we may assume the author either refers to the same argument as [9] and [10], or that the small parameter is the perturbation parameter of [7], which is equivalent to $\left\|D_{i n v}\right\|$. In any case, we believe that there is no general argument that allows replacing the average rate with the rate of the average in a nonlinear setting that does not rely upon approximately flat profiles.

The purpose here is not general criticism of the Thiele modulus approach, especially given its importance as the only alternative to the computational burden and complexity of a full $1+1 \mathrm{D}$ numerical solution in other applications in which $\left\|D_{\text {inv }}\right\|$ is not small and the washcoat concentration profiles exhibit sharp gradients in $x$. However, without awareness of at least some of the compromises and additional assumptions required when working with the Thiele modulus, it is difficult to appreciate the value of avoiding any reliance on rate constants. Because the asymptotic analysis of this paper is indifferent to any structure internal to the reaction rates, it is applicable to every reaction system with the complexity of those routinely solved by advanced commercial software without pore diffusion resistance.

The list in Table 1 demonstrates that the scopes of the two asymptotic regimes are distinct, although results will agree in the intersection of both sets of assumptions, which at minimum includes single species linear rates with an $O(1)$ rate constant and $\left\|D_{\text {inv }}\right\| \ll 1$. Since the single species linear rate case has been studied so exhaustively, we expect any results derived in the nonlinear generality of this paper will have their precedents in the linear subcase. For example, when Eqs. (14) and (15) are used to form the following ratio, the rate multipliers cancel in the numerator and denominator so that

$$
\frac{-\frac{\partial \omega_{i}}{\partial x}(0)}{\omega_{s, i}-\bar{\omega}_{i}}=3+O\left(\left\|D_{i n v}\right\|\right) .
$$

The left-hand side of this equation is defined as the internal Sherwood number (Eq. (38) of [8]) in the dimensionless variables of this paper. Since the first Aris number is $1 / 3$ for this geometry (again, see [8]), Eq. (37) extends this result, previously derived for linear reaction rates and $\phi^{2} \ll 1$ in Eq. (53) of [8], to nonlinear rates. Other such generalizations may be compared to earlier linear results, but Eq. (37) is particularly of interest because of its connection to the internal Sherwood number used in a number of the referenced papers and others.

A traditional advantage of the Thiele modulus analysis is the ability to capture completely and simultaneously the effects of both reaction and diffusion variation in the governing equations $\left(D_{i n v} R\right.$ in Eq. (5)). However, this is true only for the case of Dirichlet boundary conditions. For the more relevant mixed boundary condition, the external mass transfer coefficient, $K$, enters, and diffusion affects the governing equations importantly and independently of the species rates $\left(D_{i n v} K\right.$ in Eq. (4)), resulting in distinct reactor behavior. Again, to aid comparison between the approaches, we consider here the single reactant linear rate case. To accompany the Thiele modulus, it is conventional to use the Biot number ( $B i \equiv D_{i n v} K$ in these dimensionless variables) in the problem formulation so that Eq. (9) becomes

$B i\left(\omega_{g}-\omega_{s}\right)=\phi^{2} \bar{\omega}$.

As an example of some independent interest, we discuss briefly the transition between operating regimes, starting with kinetic control for low temperature and progressing to external mass transfer control as the temperature increases. This is a common exercise in the literature, for example in [11] or in Fig. 3.4 of [4]. The major effect of temperature is on the rate constant, so the Thiele modulus carries the effect of temperature, and the transition is from kinetic control when $\omega_{g} \approx \omega_{s} \approx \bar{\omega}$ for $\phi^{2} \ll 1$, to external mass transfer control when $\omega_{g} \gg \omega_{s} \gg \bar{\omega}$ for $\phi^{2} \gg 1$. The point here is that these transitions via Thiele modulus take qualitatively different paths depending on the magnitude of the Biot number. The Thiele modulus alone is not sufficient. For example, when $B i=O(1)$, the transition is through an intermediate regime in which $\phi^{2}$ and all the species concentrations in Eq. (38) are $O(1)$ and none of the species concentrations approximate each other: $\omega_{g}>\omega_{s}>\bar{\omega}$. When $B i \gg 1$, and the transition again passes through $\phi$ and $\bar{\omega}=O(1)$, the intermediate regime now obeys $\omega_{g} \approx \omega_{s}>\bar{\omega}$, consistent with Eq. (38). This Biot number regime is the one considered in the discussion surrounding Fig. 2 of [11], ${ }^{2}$ where the transitional regime is associated with washcoat diffusion control. Finally, in the case of interest here, $B i=D_{i n v} K \ll 1$, there are two distinguished limits involved in reaching $\phi^{2}=O(1)$. First, when $\phi^{2}=O(B i)$ and $\bar{\omega}=O(1)$, there is a regime in which $\omega_{g}>\omega_{s} \approx \bar{\omega}$, consistent with Eq. (38). As $\phi^{2}$ increases to $O(1)$ and $\bar{\omega}$ necessarily decreases to $O(B i)$ to remain consistent with Eq. (38), there is another regime in which $\omega_{g} \gg \omega_{s}>\bar{\omega}$. All these properties of the solution in this discussion can be directly verified using elementary asymptotics of the exact solution for this simple problem, given for example as Eq. (3.18) in [4].

To discuss the regimes described in [11] in slightly more detail, the intermediate washcoat diffusion control regime is described there as obeying $\omega_{g} \approx \omega_{s} \gg \bar{\omega}$ in the notation here. This can only occur in the $B i \gg 1$ case and further requires $1 \ll \phi \ll B i$.

\footnotetext{
$\overline{2}$ This is slightly different from the labeling used in [11]. See discussion in the following paragraph.
} 
We find it slightly more relevant to focus on the distinguished limit $\phi=O(1)$, for which $\omega_{g} \approx \omega_{s}>\bar{\omega}$. Since the analysis in [11] uses $B i=D_{i n v} K \gg 1$, at least for the purposes of its Fig. 2, these authors presume dominance of washcoat pore diffusion resistance to external mass transfer, consistent with their assumed values for washcoat properties later in their paper. Obviously, the regime $B i \gg 1$ is distinct from $B i \ll 1$ assumed in the present paper. We believe the latter is more representative of the thin washcoats and high effectiveness required in practical aftertreatment reactors as discussed earlier in the Discussion section. The authors of [11] recognize the distinction between these regimes in the first paragraph of their Section 5.1.

\subsection{Magnitude of Species Rates}

To summarize, $\left\|D_{i n v}\right\| \ll 1$ is a restrictive but powerful assumption, distinct but partially overlapping with $\phi^{2} \ll 1$, that allows explicit solution of the washcoat diffusion-reaction system for general, fully-coupled, nonlinear rates. Much of the advantage of this approach is in foregoing the additional assumptions required in casting the species rates into a form suitable for extracting the rate "constants" necessary for the Thiele modulus. The only requirement on the rates is $R(\omega)=O(1)$. Although this may appear to seriously constrain the magnitudes of the species rates, the overall reactant rate balance equation, Eq. (16) (or Eq. (33) for dual layers) enforces an upper bound on the net rate of any reactant of $K_{i} \omega_{g, i}$, the maximum external mass transfer rate. The overall flow properties of the reactor are used to ensure $K=O(1) . \omega_{g, i}$ provides the most representative concentration scale for the local diffusion-reaction problem, including the mixed boundary condition, as recognized by Aris in [4] in his scaling. So for the purpose of obtaining an upper bound on the local reaction rate, $\omega_{g, i}=O(1)$, and the bound $R(\omega)=O(1)$ follows. The simpler case of kinetic control, when $\omega_{s, i} \approx \omega_{g, i}$ and $\bar{R}_{i}$ is small, is a straightforward sublimit easily accommodated by the solution. For products, $\bar{R}_{i}=O(1)$ follows from the reactant species rates and stoichiometric consistency.

\subsection{If Thiele Modulus Is Not Small}

The asymptotic limit, $\left\|D_{i n v}\right\| \ll 1$, of this paper implies $B i_{i}=D_{i n v, i} K_{i} \ll 1$ and $\phi_{i}^{2}=-D_{i n v i,} k_{i} \ll 1$ since all the other dimensionless parameters of the problem statement in Section 2.1 are formally assumed $O(1)$. This includes the rate constant, which we can define with minimal assumptions (for example, reactants only) as

$k_{i}=\bar{R}_{i} / \bar{\omega}_{i}$

Necessarily then, the asymptotic solution with $\left\|D_{\text {inv }}\right\| \ll 1$ cannot cover all possible solution regimes, not even all regimes of $\phi$ when $B i \ll 1$.
As temperatures rise and the Thiele approaches $O(1)$ or larger, we now discuss the deterioration of the asymptotic solution and its impact on overall reactor performance. Comparison with the solution in Eq. (14) (or Eqs. (31) and (32) for dual layers) demonstrates that $\phi_{i}^{2}$ measures the magnitude of the ratio of the first-order spatial variations to the zeroth-order averages of the concentrations. Therefore, $\phi_{i}^{2}=O\left(\left\|D_{\text {inv }}\right\|\right)$ implies the proper ordering of the asymptotic terms. The simplest point of view would be to declare the higher limit $\phi_{i}^{2} \rightarrow O(1)$ outside the scope of the asymptotics, but in practice, it is not necessary to be that restrictive. $\phi_{i}^{2} \rightarrow O(1)$ must be obtained by $k_{i} \rightarrow O\left(1 /\left\|D_{i n v}\right\|\right)$, by definition. Since the overall rate balance will enforce $\bar{R}_{i}=O(1)$, as argued above, the limit of $\phi_{i}^{2} \rightarrow$ $O(1)$ must require $\bar{\omega}_{i} \rightarrow O\left(\left\|D_{\text {inv }}\right\|\right)$ according to Eq. (39). That is, the spatially-varying portion of the solution in Eq. (14) will still remain $O\left(\left\|D_{i n v}\right\|\right)$ as the Thiele modulus increases, but its constant portion, $\bar{\omega}_{i}$, will become small of the same order. When this occurs, the more general result involving the averages, Eq. (12) still holds, but the weaker point-wise result, Eq. (13) does not because of large $k_{i}$. Therefore, as $\phi_{i}^{2} \rightarrow O(1)$, Eq. (14) fails to capture the full leading-order solution of the reactiondiffusion equation. However, particularly for the case here of $B i \ll 1$ in contrast to larger $B i$, as $\phi_{i}^{2} \rightarrow O(1)$ the washcoat profiles stay flat and $\omega_{s, i} \rightarrow O\left(\left\|D_{i n v}\right\|\right)$ also. This is the key point because small $\omega_{s, i}$ represents the case of external mass transport limitation in which $\omega_{s, i}$ is negligible compared to $\omega_{g, i}$ in the channel gas Eq. (3). Once this limit is reached, the channel gas equation can be axially integrated without significant influence of the concentration within the washcoat. Specifically, reactor performance, in the sense of predicting $\omega_{g}(z=1)$, is not affected by the nearly zero washcoat concentrations. That is, just as the Thiele modulus becomes large enough to compromise the washcoat profiles, the surface concentration becomes so small that the channel gas equations can be calculated in the mass transport limited regime where the washcoat profile is insignificant.

Even in these cases where the washcoat concentrations are too small to influence the corresponding channel gas concentrations because of mass transport limitation, we would like to maintain various physically reasonable properties of the washcoat concentrations such as nonnegativity. While nonnegativity cannot be assured in all cases from Eqs. (31) and (32) for very large rate constants, there are modifications to the profiles that can preserve nonnegativity with slight effect otherwise. As these modifications are not essentially part of the asymptotic solution and still under evaluation, we leave this detail outside the scope of the paper.

\subsection{General Discussion}

It is worthwhile to reiterate and emphasize that the motivation and starting point for this asymptotic analysis is the case of no diffusion resistance and constant concentrations through the 
washcoat. To routinely calculate solutions even in this limiting case for the large complex reaction schemes of dozens of reactions, including site coverages, and dozens of species of both reactants and products that are common for today's practical aftertreatment reactors, it is essential to use the given reaction rates in their full nonlinear complexity and interdependence. For the applications we have in mind here, it is not practical to analyze each application in detail to justify linearizations, lumpings, selective decouplings, and so forth. The derivation given in this paper shows that when solutions are generalized to include the leading-order terms for small diffusion resistance, this essential ability to use the full nonlinear complexity is preserved. In the overall rate balance, Eq. (30), the rates are evaluated without further approximation at $\bar{\omega}^{(i)}$ rather than $\omega_{s}$, but otherwise, these rates are evaluated just as the modeling community has come to expect for no diffusion resistance. The essential recognition here is that in this asymptotic limit, the reaction terms in the reaction-diffusion system can be evaluated to leading order as a constant, and so the system has a simple explicit solution as a function of $x$, regardless of the complexity of the reaction network. Note that we are not just assuming that the reaction term can be approximated by a constant; we are identifying the physically meaningful regime, $\left\|D_{i n v}\right\| \ll 1$, where this approximation applies. By contrast, even modestly general approaches resting on the Thiele modulus, which requires an effective rate constant rather than the rate itself, ultimately must borrow results from some approximating linear problem to restore the rate from the rate constant.

Our intention is to address practical cases, where the standard solution is nearly tolerable already, which means that the gradients in the washcoat are not large. In the absence of definitive or a priori values for effective diffusivity or rate constants, our confidence in this approach is bolstered by the long-standing success of the standard solution and the belief that washcoats with poor diffusion resistance characteristics are of little interest and will be rejected for practical applications.

\section{Quantitative Comparisons}

\subsection{With 1+1D Calculation}

In this section, we test the quantitative accuracy of the asymptotic solution by comparing it to a 1+1D calculation, and we demonstrate how the quantitative accuracy gradually degrades as the pore diffusion resistance increases. The test case is a reactor with a single layer catalyst for $\mathrm{CO}$ oxidation in excess $\mathrm{O}_{2}$, as would occur in a diesel oxidation catalyst. For simplicity, most parameters were taken constant and are listed in Table 2. Conditions and the solution are steady. The single trace species for $\mathrm{CO}$ and
Table 2 Parameters used in comparison of asymptotic and numerical $1+1 \mathrm{D}$ solutions

\begin{tabular}{lll}
\hline Description & Value & Units \\
\hline Face area & 0.02 & $\mathrm{~m}^{2}$ \\
Length & 0.16 & $\mathrm{~m}$ \\
Cell density & $6.2 \times 10^{5}$ & $1 / \mathrm{m}^{2}$ \\
Substrate thickness & $1.5 \times 10^{-4}$ & $\mathrm{~m}$ \\
Washcoat thickness & $3 \times 10^{-5}$ & $\mathrm{~m}$ \\
Inlet flow rate & 0.031 & $\mathrm{~kg} / \mathrm{s}$ \\
Inlet temperature & 500 & $\mathrm{~K}$ \\
Inlet CO mole fraction & 0.001 & \\
Inlet O ${ }_{2}$ mole fraction & 0.1 & \\
Gas density & 0.77 & $\mathrm{~kg} / \mathrm{m}^{3}$ \\
Gas specific heat & $1.06 \times 10^{3}$ & $\mathrm{~J} /(\mathrm{kg} \mathrm{K})$ \\
Gas thermal conductivity & 0.036 & $\mathrm{~J} /(\mathrm{s} \mathrm{m} \mathrm{K})$ \\
Gas diffusivity & $4.5 \times 10^{-5}$ & $\mathrm{~m} / \mathrm{s}$ \\
Sherwood, Nusselt number for & 3.0 & \\
$\quad$ channel/wall transfer & & $\mathrm{J} /(\mathrm{s} \mathrm{m} \mathrm{K})$ \\
Effective axial thermal conductivity & 0.98 & $\mathrm{~J} / \mathrm{mol}$ \\
$\quad$ in wall & $2.75 \times 10^{5}$ &
\end{tabular}

the temperatures in both the gas and solid phases were solved. The dimensional rate expression [12] as a function of concentration and dimensional temperature is

$$
r=3.55 \times 10^{10} e^{-9782 / T} \frac{c_{C O} c_{O 2}}{\left(1+248 e^{-615 / T} c_{C O}\right)^{2}}, \quad\left[\frac{\mathrm{mol}}{\mathrm{m}^{3} \mathrm{~s}}\right]
$$

where $\mathrm{m}^{3}$ in the units refers to the volume of the reactor. In Fig. 1, we show several washcoat profile comparisons for different $D_{\text {eff. }}$. For the highest $D_{\text {eff }}$, the profiles are nearly indistinguishable and slowly decrease in accuracy as $D_{\text {eff }}$ decreases. In our experience so far, and not only for the simple case in this subsection, the asymptotic solution has proven to be quantitatively reliable for the realistic range $D_{\text {eff }} \geq 10^{-6} \mathrm{~m}^{2} /$ $\mathrm{s}$, with deviations increasingly noticeable below this. The solution degrades gradually so that it can still be useful at smaller $D_{\text {eff }}$, as is evident in this figure. This is the reason for displaying results for these particular values of $D_{\text {eff }}$.

To further test the impact of the asymptotic solution, we generated steady-state light-off curves of conversion against inlet temperature for both the asymptotic and 1+1D solution for these same three effective diffusivities. For the two larger values, $D_{\text {eff }}=3.33 \times 10^{-6}$ and $1 \times 10^{-6} \mathrm{~m}^{2} / \mathrm{s}$, the light-off curves from the two methods are essentially indistinguishable. For the smallest value, $D_{\text {eff }}=3.33 \times 10^{-7} \mathrm{~m}^{2} / \mathrm{s}$, a small deviation is evident at the higher temperatures, although it requires expanding this portion of the curve to see clearly in Fig. 2. This deviation arises simply because the assumption of small pore diffusion resistance is here deteriorating enough to cause 


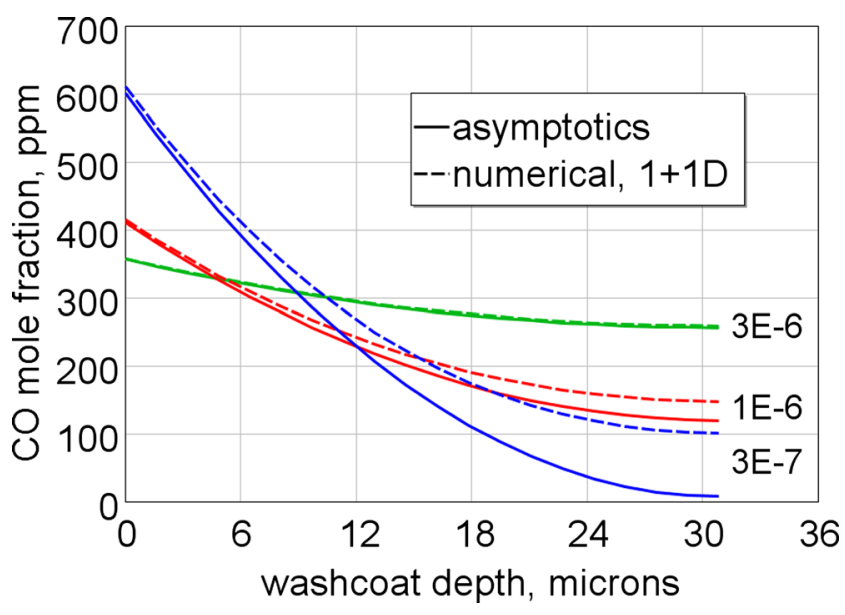

Fig. 1 Comparison of asymptotic and numerical 1+1D solutions for washcoat effective diffusivities, $D_{\text {eff }}=3.33 \times 10^{-6}, 1 \times 10^{-6}, 3.33 \times$ $10^{-7} \mathrm{~m}^{2} / \mathrm{s}$. Profiles shown at the axial location $1 / 10$ of the reactor length from the front face

small errors in the surface concentration, of the order of $10 \mathrm{ppm}$, which then have a cumulative effect on the channel gas to produce the observed small error in the conversion. Indeed, Fig. 1 shows an error in $\omega_{s}$ at $x=0.1$ of about $10 \mathrm{ppm}$ at this temperature, but this error varies axially and is nowhere greater than $30 \mathrm{ppm}$ for this case.

\subsection{With Literature Data}

To challenge our method with a complex set of reactions for realistic data, we compared our computations with experimental data found in Figure 5 of [13], which may be consulted for specifics of the application. Our purpose here is simply to exercise the implementation of our asymptotic solution on a nontrivial realistic application, not to revisit the application itself. Briefly then, a $\mathrm{Pt} / \mathrm{Al}_{2} \mathrm{O}_{3}$ ammonia oxidation catalyst was exposed to inlet gas of $300 \mathrm{ppm} \mathrm{NH}_{3}$ and excess $\mathrm{O}_{2}$ while its temperature was ramped. $\mathrm{NH}_{3}$ conversion and selectivity

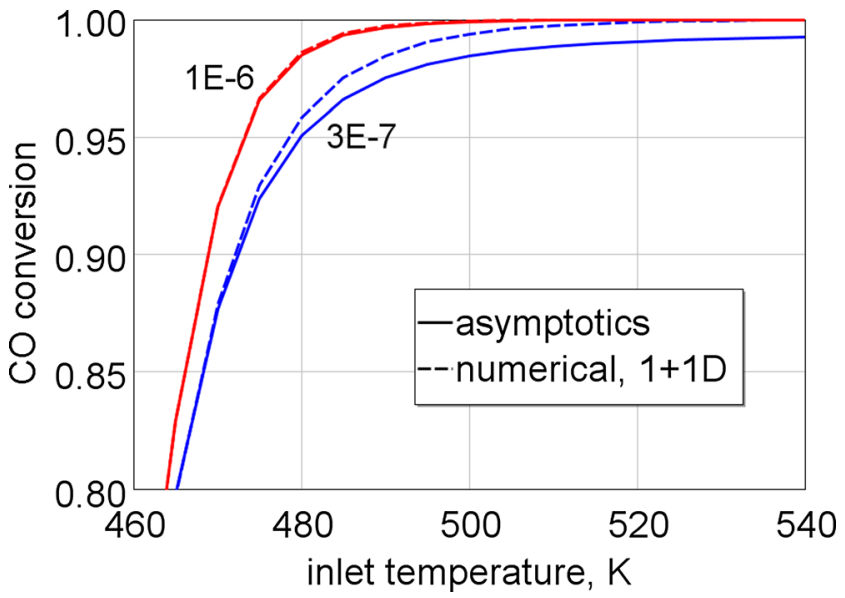

Fig. 2 Steady-state CO conversions for asymptotic and numerical 1+1D solutions for washcoat effective diffusivities, $D_{\text {eff }}=1 \times 10^{-6}$ and $3.33 \times$ $10^{-7} \mathrm{~m}^{2} / \mathrm{s}$. Only portions where differences are evident are shown toward the various $\mathrm{N}$ species was measured. Then, a second layer of selective catalytic reduction (SCR) catalyst was added on top, and these measurements were repeated to study the effect of the dual layer. Modeling this experiment requires integration of the washcoat reaction-diffusion solution within the reactor model at all axial locations and times. The authors in [13] note the existence and importance of concentration gradients within the washcoats from their own computations and conclude that the $\mathrm{NH}_{3}$ conversion suffered from the second layer because of the additional diffusion resistance of the added layer. On the other hand, the selectivity improved, as desired.

To model the reactions in the Pt layer, we used a 5-step global mechanism calibrated to the data in Figure 5 of [13]. For the SCR layer, we used a 2-site, 11-step global mechanism that we had previously calibrated for a different application. Both reaction mechanisms are provided in Appendix 2. Our calculations were transient, based on the slow temperature ramp rates used in the experiments, and included the energy
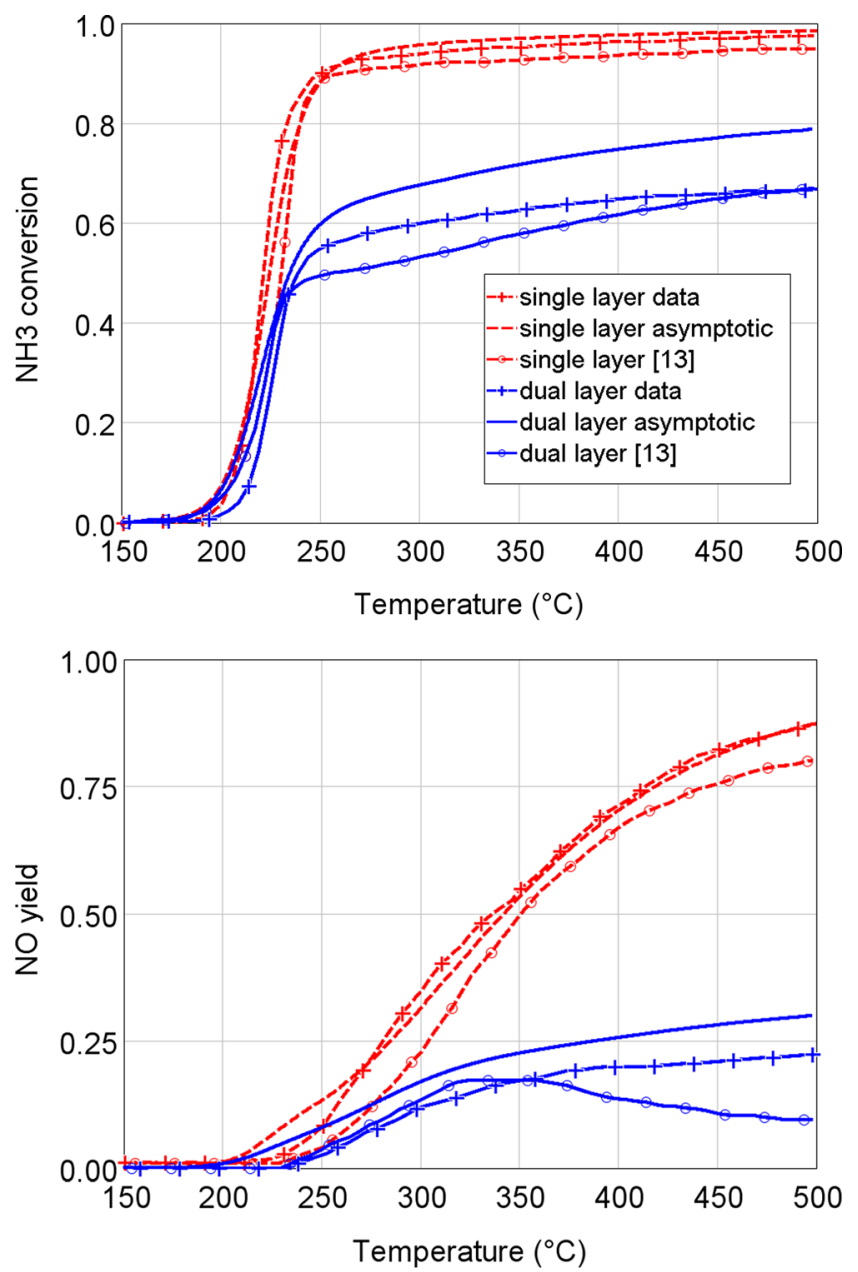

Fig. 3 Selected asymptotic results to compare with Figure 5 in [13]. Curves with symbols are from [13]: plus sign for data and circle for computations in [13]. Curves without symbols are from the asymptotic methods of the present paper 
equation. Sample results for $\mathrm{NH}_{3}$ and $\mathrm{NO}$ are shown in Fig. 3 . These model predictions (and also the species not shown) compare favorably with the data in [13], with agreement comparable to the computations of the original authors. On a standard desktop computer, the single layer calculation took about $5 \mathrm{~s}$ and the dual layer calculation took about $10 \mathrm{~s}$ using our current implementation in GT-Power.

Acknowledgments I thank all the members of the Aftertreatment Group at Gamma Technologies for their advice and support. In particular, I am grateful to Ryan Dudgeon for supplying the comparisons with literature data and especially for steadfastly checking my implementations and to Dr. Santhosh Gundlapally for providing the 1+1D calculations along with their comparisons.

\section{Appendix 1 Quantitative Estimate of $D_{\text {eff }}$}

$D_{i n v}$ is the inverse matrix of the dimensionless effective washcoat pore diffusivities.

$D_{i n v}=\left[D_{e f f} / D_{r}\right]^{-1}$,

where $D_{\text {eff }}$ is the diagonal matrix of dimensional $\left(\mathrm{m}^{2} / \mathrm{s}\right)$ effective diffusivities. The constant reference diffusivity, $D_{r}$, will involve the natural length scale for pore diffusion, $\delta$, and other quantities consistent with the remainder of the "standard" problem. We do not want to imply that this approach is linked to any particular nondimensionalization, which may introduce a degree of individual judgment and preference. Our goal in scaling/nondimensionalization is to achieve roughly $O(1)$ dimensionless quantities for the underlying "standard" problem. The choice to scale the distance through the washcoat by $\delta$ will then determine $D_{r}$, which in turn will determine the magnitude of $\left\|D_{i n v}\right\|$. By using this norm as the small parameter, we implicitly assume by $\left\|D_{i n v}\right\| \ll 1$ that all the dimensionless effective diffusion resistances are small.

In our work, each species mass fraction is scaled by an application-specific reference quantity, $\omega_{r, i}$, and the species rates are nondimensionalized by $\omega_{r, i} W_{r} / V,\left(\mathrm{~kg} /\left(\mathrm{m}^{3} \mathrm{~s}\right)\right)$. This, along with the scaling by $\delta$ to obtain $x$ will yield

$D_{r}=\frac{\delta^{2} W_{r}}{\rho_{s} f_{w c} V}$

To roughly estimate for typical applications, we take $W_{r} /$ $\left(\rho_{s} V\right) \approx 2 \times(S V) \approx 15 \mathrm{~s}^{-1}$, where the space velocity at standard conditions $(S V)$ was taken at $27,000 \mathrm{~h}^{-1}$, and the factor of 2 is to correct to higher temperatures. For square channels, we estimate $\delta^{2} / f_{w c} \approx D_{h} \delta / 4 \approx 7.5 \times 10^{-9}\left(\mathrm{~m}^{2}\right)$, where we have taken the hydraulic diameter, $D_{h}$, of $1 \mathrm{~mm}$, and washcoat thickness of $30 \mu \mathrm{m}$. The resulting $D_{r} \approx 1.1 \times 10^{-7}$ is sufficiently small compared to common estimates of $D_{\text {eff }}$ for mainstream applications to justify claims of $\left\|D_{i n v}\right\| \ll 1$ in the main text.
Appendix 2 Kinetic Details for Comparison of Section 6.2

For the comparison with the data in [13], we used a global reaction mechanism below. Temperature, $T$, is $\mathrm{K}$; pressure, $p$, is $\mathrm{Pa}$; concentrations, $c_{i}$, are $\mathrm{mol} / \mathrm{m}^{3}$; and resulting rates are turnover numbers, $\mathrm{mol} /(\mathrm{s}$ mol-sites).

For $\mathrm{NH}_{3}$ oxidation in the Pt layer:

\begin{tabular}{|c|c|}
\hline \multicolumn{2}{|c|}{$2 \mathrm{NH}_{3}+1.5 \mathrm{O}_{2} \rightarrow \mathrm{N}_{2}+3 \mathrm{H}_{2} \mathrm{O}$} \\
\hline \multicolumn{2}{|c|}{$2 \mathrm{NH}_{3}+2.5 \mathrm{O}_{2} \rightarrow 2 \mathrm{NO}+3 \mathrm{H}_{2} \mathrm{O}$} \\
\hline \multicolumn{2}{|c|}{$2 \mathrm{NH}_{3}+2 \mathrm{NO}+1.5 \mathrm{O}_{2} \rightarrow 2 \mathrm{~N}_{2} \mathrm{O}+3 \mathrm{H}_{2} \mathrm{O}$} \\
\hline $\mathrm{NO}+0.5 \mathrm{O}_{2} \leftrightarrow \mathrm{NO}_{2}$ & $\begin{array}{l}1.115 \times 10^{7} e^{-5250 / T} \\
\quad\left(c_{N O} \sqrt{c_{O 2}}-c_{N O 2} G_{2}\right) / G_{1}\end{array}$ \\
\hline $\begin{array}{l}2 \mathrm{NH}_{3}+2 \mathrm{NO}_{2} \rightarrow \mathrm{N}_{2}+\mathrm{N}_{2} \mathrm{O}+ \\
\quad 3 \mathrm{H}_{2} \mathrm{O}\end{array}$ & $6.27 \times 10^{13} e^{-6530 / T} c_{N H 3} c_{N O 2}$ \\
\hline \multirow{2}{*}{\multicolumn{2}{|c|}{$\begin{array}{l}\text { where } G_{1}=1+3.30 \times 10^{6} e^{-1327 / T} c_{N O 2} \text { and } G_{2}=7.36 \times \\
10^{5} e^{-6860 / T} / \sqrt{T} \\
\text { For the SCR layer, there are two } \mathrm{NH}_{3} \text { storage sites, } \mathrm{S} 1 \text { and } \\
\mathrm{S} 2 \text {, with } \mathrm{NH}_{3} \text { coverages } \theta_{1} \text { and } \theta_{2} \text {, and corresponding "va- } \\
\text { cant" coverages } \theta_{v 1} \text { and } \theta_{v 2} \text {. }\end{array}$}} \\
\hline & \\
\hline $\mathrm{S} 1+\mathrm{NH} 3 \leftrightarrow \mathrm{Z} 1 \mathrm{NH} 3$ & $0.657 c_{N H 3} \theta_{v 1}-13.25 e^{-4760\left(1-.89 \theta_{1}\right) / T} \theta_{1}$ \\
\hline $\begin{array}{l}4 \mathrm{NH}_{3}-\mathrm{S} 1+3 \mathrm{O}_{2} \\
\quad \rightarrow 2 \mathrm{~N}_{2}+6 \mathrm{H}_{2} \mathrm{O}+4 \mathrm{~S} 1\end{array}$ & $8.77 \times 10^{8} e^{-21400 / T} \sqrt{c_{O 2} /(p T)} \theta_{1}$ \\
\hline $\begin{array}{l}4 \mathrm{NH}_{3}-\mathrm{S} 1+4 \mathrm{NO}+\mathrm{O}_{2} \\
\quad \rightarrow 4 \mathrm{~N}_{2}+6 \mathrm{H}_{2} \mathrm{O}+4 \mathrm{~S} 1\end{array}$ & $4.87 \times 10^{5} e^{-5860 / T} c_{N O} \sqrt{c_{O 2} /(p T)} \frac{\theta_{v 1} \theta_{1}}{\theta_{v 1}+52.3 \theta_{1}}$ \\
\hline $\mathrm{NO}+0.5 \mathrm{O}_{2} \leftrightarrow \mathrm{NO}_{2}$ & $56.3 e^{-3730 / T}\left(c_{\mathrm{NO}} \sqrt{c_{\mathrm{O} 2}}-c_{\mathrm{NO} 2} G_{2}\right)$ \\
\hline $\begin{array}{l}4 \mathrm{NH}_{3}-\mathrm{S} 1+2 \mathrm{NO}+2 \mathrm{NO}_{2} \\
\rightarrow 4 \mathrm{~N}_{2}+6 \mathrm{H}_{2} \mathrm{O}+4 \mathrm{~S} 1\end{array}$ & $1.53 \times 10^{15} e^{-13600 / T} c_{N O} c_{N O 2} \theta_{1}$ \\
\hline $\begin{array}{l}8 \mathrm{NH}_{3}-\mathrm{S} 1+6 \mathrm{NO}_{2} \\
\rightarrow 7 \mathrm{~N}_{2}+12 \mathrm{H}_{2} \mathrm{O}+8 \mathrm{~S} 1\end{array}$ & $7.28 \times 10^{11} e^{-15100 / T} c_{N O 2} \theta_{1}$ \\
\hline $\mathrm{S} 2+\mathrm{NH}_{3} \leftrightarrow \mathrm{NH}_{3}-\mathrm{S} 2$ & $40 c_{N H 3} \theta_{v 2}-1.973 \times 10^{7} e^{-11090 / T} \theta_{2}$ \\
\hline $\begin{array}{l}4 \mathrm{NH}_{3}-\mathrm{S} 2+3 \mathrm{O}_{2} \\
\quad \rightarrow 2 \mathrm{~N}_{2}+6 \mathrm{H}_{2} \mathrm{O}+4 \mathrm{~S} 2\end{array}$ & $8.77 \times 10^{8} e^{-21400 / T} \sqrt{c_{O 2} /(p T)} \theta_{2}$ \\
\hline $\begin{array}{l}4 \mathrm{NH}_{3}-\mathrm{S} 2+4 \mathrm{NO}+\mathrm{O}_{2} \\
\quad \rightarrow 4 \mathrm{~N}_{2}+6 \mathrm{H}_{2} \mathrm{O}+4 \mathrm{~S} 2\end{array}$ & $2.91 \times 10^{6} e^{-5860 / T} c_{N O} \sqrt{c_{O 2} /(p T)} \frac{\theta_{v 2} \theta_{2}}{\theta_{v 2}+52.3 \theta_{2}}$ \\
\hline $\begin{array}{l}4 \mathrm{NH}_{3}-\mathrm{S} 2+2 \mathrm{NO}+2 \mathrm{NO}_{2} \\
\rightarrow 4 \mathrm{~N}_{2}+6 \mathrm{H}_{2} \mathrm{O}+4 \mathrm{~S} 2\end{array}$ & $1.53 \times 10^{15} e^{-13600 / T} c_{\mathrm{NO} c_{\mathrm{NO} 2}} \theta_{2}$ \\
\hline $\begin{array}{l}8 \mathrm{NH}_{3}-\mathrm{S} 2+6 \mathrm{NO}_{2} \\
\quad \rightarrow 7 \mathrm{~N}_{2}+12 \mathrm{H}_{2} \mathrm{O}+8 \mathrm{~S} 2\end{array}$ & $7.28 \times 10^{11} e^{-15100 / T} c_{N O 2} \theta_{2}$ \\
\hline
\end{tabular}

The dispersion in the Pt layer was $15 \%$. Active site densities in the SCR layer were $115 \mathrm{~mol} / \mathrm{m}^{3}$ for S1 and $25 \mathrm{~mol} / \mathrm{m}^{3}$ for $\mathrm{S} 2$.

Also, to scale up this laboratory scale reactor closer to the range for applications, we increased the reactor volume by a factor of 100 , maintaining the normalized space velocity.

\section{References}

1. Bender, C.M., Orszag, S.A.: Advanced mathematical methods for scientists and engineers, p. 124. McGraw-Hill, New York (1978) 
2. Hayes, R.E., Kolaczkowski, S.T., Li, P.K.C., Awdry, S.: Evaluating the effective diffusivity of methane in the washcoat of a honeycomb monolith. Appl. Catal. B: Environ. 25, 93-104 (2000)

3. Zhang, F., Hayes, R.E., Kolaczkowski, S.T.: A new technique to measure the effective diffusivity in a catalytic monolith washcoat. Chem. Eng. Res. Des. 82(A4), 481-489 (2004)

4. Aris, R.: The mathematical theory of diffusion and reaction in permeable catalysts, volume I, the theory of the steady state. Oxford University Press, London (1975)

5. Kumar, P., Gu, T., Grigoriadis, K., Franchek, M., Balakotaiah, V.: Spatio-temporal dynamics of oxygen storage and release in a three-way catalytic converter. Chem. Eng. Sci. 111, 180-190 (2014)

6. Mladenov, N., Koop, J., Tischer, S., Deutschmann, O.: Modeling of transport and chemistry in channel flows of automotive catalytic converters. Chem. Eng. Sci. 65, 812-826 (2010)

7. Chakraborty, S., Balakotaiah, V.: Spatially averaged multiscale models for chemical reactors. Adv. Chem. Eng. 30, 205-297 (2005)
8. Balakotaiah, V.: On the relationship between Aris and Sherwood numbers and friction and effectiveness factors. Chem. Eng. Sci. 63, 5802-5812 (2008)

9. Joshi, S.Y., Harold, M.P., Balakotaiah, V.: Low-dimensional models for real time simulations of catalytic monoliths. Am Inst. Chem. Eng. J. 55(7), 1771-1783 (2009)

10. Joshi, S.Y., Harold, M.P., Balakotaiah, V.: On the use of internal mass transfer coefficients in modeling of diffusion and reaction in catalytic monoliths. Chem. Eng. Sci. 64, 4976-4991 (2009)

11. Joshi, S.Y., Harold, M.P., Balakotaiah, V.: Overall mass transfer coefficients and controlling regimes in catalytic monoliths. Chem. Eng. Sci. 65, 1729-1747 (2010)

12. Sampara, C.S., Bissett, E.J., Chmielewski, M.: Global Kinetics for a commercial diesel oxidation catalyst with two exhaust hydrocarbons. Ind. Eng. Chem. Res. 47, 311-322 (2008)

13. Scheuer, A., Hauptmann, W., Drochner, A., Gieshoff, J., Vogel, H., Votsmeier, M.: Dual layer automotive ammonia oxidation catalysts: experiments and computer simulation. Appl. Catal. B Environ. 111112, 445-455 (2012) 